\title{
The volume near the zeroes of a smooth function
}

\section{Pavel Batchourine and Charles Fefferman}

\begin{abstract}
We show that if a smooth function that never vanishes to infinite order, then the set of points within the distance $\delta$ from the zeroes of this function has volume $O(\delta)$.
\end{abstract}

\section{Statement of Result}

Let $B(x, r)$ denote the open ball of radius $r$ about $x$ in $\mathbb{R}^{n}$. In this note we prove the following result.

Theorem 1. Let $F$ be a real-valued $C^{m}$ function on $B(0,1)$, with

1. $c_{0}<\max _{|\alpha|=m-1}\left|\partial^{\alpha} F(0)\right|<C_{0}$, and with

2. $\left|\partial^{\alpha} F\right| \leq C_{1}$ on $B(0,1)$ for $|\alpha|=m$.

Let

3. $V(F)=\{x \in B(0,1): F(x)=0\}$, and let

4. $V(F, \delta)=\left\{x \in B\left(0, c_{1}\right):\right.$ distance $\left.(x, V(F))<\delta\right\}$,

where $c_{1}$ is a small enough constant determined by $c_{0}, C_{0}, C_{1}, m, n$.

Then we have

$$
\operatorname{Vol}\{V(F, \delta)\} \leq C_{2} \delta \text { for } 0<\delta<c_{1},
$$

where $C_{2}$ is a large constant determined by $c_{0}, C_{0}, C_{1}, m, n$.

Thus, if $F$ is a smooth function that never vanishes to infinite order, then the set of points within the distance $\delta$ from the zeroes of $F$ has volume $O(\delta)$. If we allow $F$ to vanish to infinite order then the corresponding assertion is plainly wrong. For the level sets of polynomials this statement is proven in [1]. 


\section{A Convenient Reduction}

In this section, we reduce Theorem 1 to the following result which is seemingly a bit less general.

Theorem 2. Let $F$ be a real-valued $C^{m}$ function on $B(0,1)$, with

1. $c_{0}<\left|\partial^{\alpha} F\right|<C_{0}$ everywhere on $B(0,1)$, for every multi-index $\alpha$ of order $m-1$,

2. $\left|\partial^{\alpha} F\right| \leq C_{1}$ everywhere on $B(0,1)$ for every multi-index $\alpha$ of order $m$.

Let

3. $V(F)=\{x \in B(0,1): F(x)=0\}$, and let

4. $V(F, \delta)=\left\{x \in B\left(0, c_{1}\right):\right.$ distance $\left.(x, V(F))<\delta\right\}$,

where $c_{1}$ is a small enough constant determined by $c_{0}, C_{0}, C_{1}, m, n$.

Then we have

$$
\operatorname{Vol}\{V(F, \delta)\} \leq C_{2} \delta \quad \text { for } 0<\delta<c_{1},
$$

where $C_{2}$ is a large constant determined by $c_{0}, C_{0}, C_{1}, m, n$.

To reduce Theorem 1 to Theorem 2, we use the following elementary result.

Proposition 3. Let $F$ satisfy the hypotheses of Theorem 1. Then there exists a linear map $A: \mathbb{R}^{n} \rightarrow \mathbb{R}^{n}$, and constants $c$ and $C$, with the following properties:

1. $c$ and $C$ are determined by $c_{0}, C_{0}, C_{1}, m, n$,

2. the maps $A$ and $A^{-1}$ have norms at most $C$,

3. $F \circ A$ is well-defined on $B(0, c)$,

4. $c<\left|\partial^{\alpha}(F \circ A)\right|<C$ on $B(0, c)$ for all $\alpha$ with $|\alpha|=m-1$,

5. $\left|\partial^{\alpha}(F \circ A)\right|<C$ on $B(0, c)$ for all $\alpha$ with $|\alpha|=m$.

Once the proposition is proven, then the Theorem 1 follows by applying Theorem 2 to the function $\tilde{F}(x)=(F \circ A)(c x), x \in B(0,1)$.

Proof of the Proposition. In this proof, we say that a constant is controlled, if it is determined by $c_{0}, C_{0}, C_{1}, m$ and $n$; and we write $c, C, C^{\prime}$, etc. to denote the controlled constants.

Pick a vector $v \in \mathbb{R}^{n}$ of length 1 to maximize $\left|(v \cdot \nabla)^{m-1} F(0)\right|$. Without loss of generality, we may assume that $v=e_{n}$, the n'th unit vector in $\mathbb{R}^{n}$. Then we have

$$
c<\left|\left(\frac{\partial}{\partial x_{n}}\right)^{m-1} F(0)\right|<C, \quad \text { and } \quad\left|\partial^{\alpha} F(0)\right|<C \text { for }|\alpha|=m-1 .
$$


Consequently, for any $\lambda \in(0,1)$, and for any multi-index $\alpha=\left(\alpha_{1}, \ldots \alpha_{n}\right)$ with $|\alpha|=\alpha_{1}+\ldots+\alpha_{n}=m-1$, we have

$$
\left(\frac{\partial}{\partial x_{n}}+\lambda \frac{\partial}{\partial x_{1}}\right)^{\alpha_{1}} \ldots\left(\frac{\partial}{\partial x_{n}}+\lambda \frac{\partial}{\partial x_{n-1}}\right)^{\alpha_{n-1}}\left(\frac{\partial}{\partial x_{n}}\right)^{\alpha_{n}} F(0)=\sum_{k=0}^{m-1} A_{k}^{(\alpha)} \lambda^{k}
$$

with $c<\left|A_{0}^{(\alpha)}\right|<C$ and $\left|A_{k}^{(\alpha)}\right|<C$ for all $k$.

Therefore, if we take $\lambda=\bar{c}$ for small enough controlled constant $\bar{c}$, then we obtain

$$
c<\left|\left(\frac{\partial}{\partial x_{n}}+\lambda \frac{\partial}{\partial x_{1}}\right)^{\alpha_{1}} \ldots\left(\frac{\partial}{\partial x_{n}}+\lambda \frac{\partial}{\partial x_{n-1}}\right)^{\alpha_{n-1}}\left(\frac{\partial}{\partial x_{n}}\right)^{\alpha_{n}} F(0)\right|<C
$$

for all $\alpha=\left(\alpha_{1}, \ldots \alpha_{n}\right)$ with $|\alpha|=m-1$.

We define

$$
A:\left(x_{1}, \ldots x_{n}\right) \mapsto\left(x_{n}+\lambda x_{1}, \ldots, x_{n}+\lambda x_{n-1}, x_{n}\right)
$$

Thus

$$
\|A\|,\left\|A^{-1}\right\| \leq C
$$

and

$$
c<\left|\partial^{\alpha}(F \circ A)(0)\right|<C \text { for all } \alpha \text { with }|\alpha|=m-1 .
$$

From (2.1), and from hypothesis (2) of Theorem 1, we see then

$$
F \circ A \text { is well-defined on } B(0, c), \text { and }
$$

$$
\left|\partial^{\alpha}(F \circ A)\right|<C \text { on } B(0, c), \quad \text { for all } \alpha \text { with }|\alpha|=m \text {. }
$$

From (2.2), (2.3), (2.4), we obtain

$$
c^{\prime}<\left|\partial^{\alpha}(F \circ A)\right|<C^{\prime} \text { on } B\left(0, c^{\prime \prime}\right), \quad \text { for all } \alpha \text { with }|\alpha|=m-1 \text {. }
$$

Since $c, C, c^{\prime}, C^{\prime}, c^{\prime \prime}$ are controlled constants, the conclusion of our proposition follows at once from $(2.1),(2.3),(2.4),(2.5)$. The proof of the proposition is complete.

Thus we have reduced Theorem 1 to Theorem 2 . 


\section{An Elementary Remark}

For $i=1, \ldots n$, let $e_{i}$ denote the $i$ 'th unit vector in $\mathbb{R}^{n}$. In this section we recall the following elementary result.

Proposition 4. Let $M_{1}, M_{2}, a_{1}, \delta, \Gamma$ be positive real numbers and let $G$ be a real-valued $C^{2}$ function on $B\left(x^{0}, 2 \delta\right)$. Assume that

1. $\left|\frac{\partial}{\partial x_{i}} G\right| \leq M_{1} \Gamma \delta^{-1}$ and $\left|\frac{\partial^{2}}{\partial x_{i} \partial x_{j}} G\right| \leq M_{2} \Gamma \delta^{-2}$ on $B\left(x^{0}, 2 \delta\right)$;

2. $\left|\frac{\partial}{\partial x_{i_{0}}} G\left(x^{0}\right)\right| \geq a_{1} \Gamma \delta^{-1}$ and

3. $\left|G\left(x^{0}\right)\right| \leq a_{*} \Gamma$ for all small enough $a_{*}$, determined by $M_{1}, M_{2}, a_{1}, n$.

Then, for any $x \in B\left(x^{0}, a_{*} \delta\right)$, there exists $\tau \in(-\delta, \delta)$ such that $G\left(x+\tau e_{i_{0}}\right)=0$.

Proof. By rescaling, we may suppose $\Gamma=\delta=1$. Integrating $|\nabla G|$ and $\left|\nabla \frac{\partial}{\partial x_{i_{0}}} G\right|$ on the line segment joining $x^{0}$ to $x$, we find that

$$
\begin{gathered}
\left|G(x)-G\left(x^{0}\right)\right| \leq \sqrt{n} M_{1}\left|x-x^{0}\right| \leq \sqrt{n} M_{1} a_{*}, \quad \text { and } \\
\left|\frac{\partial}{\partial x_{i_{0}}} G(x)-\frac{\partial}{\partial x_{i_{0}}} G\left(x^{0}\right)\right| \leq \sqrt{n} M_{2}\left|x-x^{0}\right| \leq \sqrt{n} M_{2} a_{*}
\end{gathered}
$$

Hence,

$$
|G(x)| \leq\left(1+\sqrt{n} M_{1}\right) a_{*}, \quad \text { and }
$$

$$
\left|\frac{\partial}{\partial x_{i_{0}}} G(x)\right| \geq a_{1}-\sqrt{n} M_{2} a_{*} \geq 1 / 2 a_{1}, \quad \text { (if we take } a_{*} \text { small enough) }
$$

Since also $\left|\left(\frac{\partial}{\partial x_{i_{0}}}\right)^{2} G\right| \leq M_{2}$ on $B\left(x^{0}, 2\right),(3.2)$ implies that

$$
\left|\frac{\partial}{\partial x_{i_{0}}} G\left(x+\tau e_{i_{0}}\right)\right| \geq 1 / 2 a_{1}-M_{2}|\tau| \geq 1 / 4 a_{1},
$$

for $\tau \in\left[-\frac{a_{1}}{4 M_{2}}, \frac{a_{1}}{4 M_{2}}\right] \cap(-1,1)=I$.

Let $g(\tau)=G\left(x+\tau e_{i_{0}}\right)$ for $\tau \in I$. Then $g$ is a $C^{2}$-function on $I$; and (3.1), (3.3) yield

$$
|g(0)| \leq\left(1+\sqrt{n} M_{1}\right) a_{*}, \quad \text { and } \quad\left|g^{\prime}\right| \geq 1 / 4 a_{1} \text { on } I
$$

If $a_{*}$ is taken small enough, then (3.4) easily implies $g(\tau)=0$ for some $\tau \in I$. In particular, $G\left(x+\tau e_{i_{0}}\right)=0$ for some $\tau \in(-1,1)$, proving the proposition. 


\section{Two Main Lemmas}

From now on, we assume that the function $F$ and the constants $c_{0}, C_{0}, C_{1}$ satisfy the hypothesis of the Theorem 2 . We say that a constant is controlled, if it is determined by $c_{0}, C_{0}, C_{1}, m$ and $n$; and we write $c, C, C^{\prime}$, etc. to denote the controlled constants.

As in the Section 2, we write $e_{1}, \ldots, e_{n}$ for the unit vectors in $\mathbb{R}^{n}$.

Lemma 5. For a small enough controlled constant $\bar{c}$, the following holds. Suppose $x^{0} \in V(F) \cap B(0,1 / 2)$, and suppose $0<\delta<\bar{c}$. Then, for any $x \in B\left(x^{0}, \bar{c} \delta\right)$, there exist $\beta, i_{0}, \tau$ with

1. $|\beta| \leq m-2,1 \leq i_{0} \leq n$;

2. $\tau \in[-\delta, \delta]$ and

3. $\partial^{\beta} F\left(x+\tau e_{i_{0}}\right)=0$.

Proof. Let $A_{m}, A_{m-1}, \ldots A_{0}$ be constants to be picked later. We write $C\left(A_{m}, \ldots A_{k}\right)$ to denote a constant determined by $A_{m}, \ldots A_{k}$ and $c_{0}, C_{0}, C_{1}$, $m, n$. We define

$$
\Omega=\max _{|\gamma| \leq m-1} A_{|\gamma|} \delta^{|\gamma|}\left|\partial^{\gamma} F\left(x^{0}\right)\right|,
$$

and we suppose that the max in (4.1) is attained at $\gamma=\bar{\gamma}$. From the hypothesis (1) of the Theorem 2, we have

$$
\Omega \geq A_{m-1} c_{0} \delta^{m-1}
$$

In particular, $\Omega \neq 0$. Since $x^{0} \in V(F)$, we have $F\left(x^{0}\right)=0$, so the maximum in (4.1) is not attained at $\gamma=0$. Hence, $\bar{\gamma} \neq 0$, and consequently, we may write $\bar{\gamma}=1_{i_{0}}+\beta$, where $|\beta| \leq m-2$, and $1_{i_{0}}$ is the $i_{0}$-th unit multi-index. In particular, $i_{0}$ and $\beta$ satisfy (1). By the definition of $\Omega, \bar{\gamma}$, $i_{0}, \beta$, we have

$$
\begin{gathered}
\left|\partial^{\gamma} F\left(x^{0}\right)\right| \leq A_{|\gamma|}^{-1} \Omega \delta^{-|\gamma|} \text { for }|\gamma| \leq m-1, \text { and } \\
\left|\frac{\partial}{\partial x_{i_{0}}}\left(\partial^{\beta} F\right)\left(x^{0}\right)\right|=A_{|\beta|+1}^{-1} \Omega \delta^{-|\beta|-1}
\end{gathered}
$$

Also, for $|\gamma|=m, x \in B(0,1)$, estimate (4.2) and the hypothesis (2) of the Theorem 2 yield

$$
\left|\partial^{\gamma} F(x)\right| \leq C_{1} \leq C_{1} c_{0}^{-1} A_{m-1}^{-1} \Omega \delta^{-(m-1)}
$$

If

$$
0<\delta<A_{m}^{-1} c_{0} A_{m-1} C_{1}^{-1}
$$

then (4.5) implies

$$
\left|\partial^{\gamma} F\right| \leq A_{m}^{-1} \Omega \delta^{-|\gamma|} \text { on } B(0,1), \text { for }|\gamma|=m .
$$


From (4.3), (4.7) and Taylor's theorem, we obtain

$$
\left|\partial^{\gamma} F\right| \leq C\left(A_{m}, \ldots A_{|\gamma|}\right) \Omega \delta^{-|\gamma|} \quad \text { on } B\left(x^{0}, 2 \delta\right), \text { for }|\gamma| \leq m,
$$

provided

$$
\delta<1 / 4
$$

(Condition (4.9) guarantees that $B\left(x^{0}, 2 \delta\right) \subset B(0,1)$, since $\left.x^{0} \in B(0,1 / 2)\right)$

In particular, (4.8) gives

$$
\left|\frac{\partial}{\partial x_{i}}\left[\partial^{\beta} F\right]\right| \leq C\left(A_{m}, \ldots, A_{|\beta|+1}\right) \Omega \delta^{-|\beta|-1} \quad \text { on } B\left(x^{0}, 2 \delta\right),
$$

and

(4.11) $\left|\frac{\partial^{2}}{\partial x_{i} \partial x_{j}}\left[\partial^{\beta} F\right]\right| \leq C\left(A_{m}, \ldots, A_{|\beta|+2}\right) \Omega \delta^{-|\beta|-2}$ on $B\left(x^{0}, 2 \delta\right)$ for all $i, j$.

Also, (4.3) and (4.4) give

$$
\left|\frac{\partial}{\partial x_{i_{0}}}\left[\partial^{\beta} F\right]\left(x^{0}\right)\right|=A_{|\beta|+1}^{-1} \Omega \delta^{-|\beta|-1}
$$

and

$$
\left|\left[\partial^{\beta} F\right]\left(x^{0}\right)\right| \leq A_{|\beta|}^{-1} \Omega \delta^{-|\beta|}
$$

Note that $A_{|\beta|}$ appears in (4.13), but not in (4.10), (4.11), (4.12). Suppose that

$$
A_{|\beta|} \text { exceeds a large enough constant } C\left(A_{m}, \ldots, A_{|\beta|+1}\right),
$$

Then (4.10)-(4.14) are the hypotheses of the proposition 3 with $G=\partial^{\beta} F$, $\Gamma=\Omega \delta^{-|\beta|}, M_{1}=C\left(A_{m}, \ldots, A_{|\beta|+1}\right), M_{2}=C\left(A_{m}, \ldots, A_{|\beta|}+2\right), a_{1}=A_{|\beta|+1}^{-1}$, $a_{*}=A_{|\beta|}^{-1}$. Applying the proposition, we learn the following:

Given $x \in B\left(x^{0}, A_{|\beta|}^{-1} \delta\right)$, there exists $\tau \in(-\delta, \delta)$,

$$
\text { such that } \partial^{\beta} F\left(x+\tau e_{i_{0}}\right)=0 \text {. }
$$

We now take $A_{m}=A_{m-1}=1$, and successively pick the controlled constants $A_{m-2}, A_{m-3}, \ldots A_{0}$, so that (4.14) holds for all $|\beta| \leq m-2$. In particular, if $\bar{c}$ is a small enough controlled constant, and if $0<\delta<\bar{c}$, then (4.6) and (4.9) are satisfied, and (4.15) applies to all $x \in B\left(x^{0}, \bar{c} \delta\right)$. Since we have already noted, that (1) holds, the conclusions of the lemma 5 are obvious from (4.15). 
From now on, we fix $\bar{c}$ as in the Lemma 5 .

We prepare to state our second Lemma. Let $0<\delta<\bar{c}$ be given. Fix a cube $Q^{0}$ centered at the origin, such that

$$
1 / 4 \leq \operatorname{diameter} Q^{0}<1 / 2,
$$

and such that diameter $Q^{0}$ is an integer multiple of $\delta$. Then we can partition $Q^{0}$ into cubes $\left\{Q_{\nu}\right\}$ of diameter $\bar{c} \delta$.

Let $x_{\nu}$ be the center of $Q_{\nu}$. Note that $Q^{0} \subset B(0,1 / 2)$, thanks to (4.16).

We define a label to be an ordered pair $\left(i_{0}, \beta\right)$ satisfying condition (1) of Lemma 5 . We say, that the cube $Q_{\nu}$ carries the label $\left(i_{0}, \beta\right)$, provided we have $\partial^{\beta} F\left(x_{\nu}+\tau e_{i_{0}}\right)=0$ for some $\tau \in[-\delta, \delta]$. From Lemma 5 (applied to $x=x_{\nu}$ ), we learn the following basic fact:

$$
\text { Every } Q_{\nu} \text { containing a zero of } F \text { must carry some label. }
$$

On the other hand, we have the following result.

Lemma 6. Fix a label $\left(i_{0}, \beta\right)$. Then there are at most $C \delta^{-(n-1)}$ cubes $Q_{\nu}$ that carry the given label.

Proof. Without loss of generality, we may suppose that $i_{0}=n$. We arrange the cubes $Q_{\nu}$ into columns", by saying that $Q_{\nu}$ and $Q_{\nu^{\prime}}$ belong to the same "column" if their centers $x_{\nu}$ and $x_{\nu^{\prime}}$ differ at most in the $n$-th coordinate. There are at most $C \delta^{-(n-1)}$ distinct columns. Hence, to prove lemma 6 , it is enough to show that any given column contains at most $C$ distinct $Q_{\nu}$ that carry the label $\left(i_{0}, \beta\right)$.

Fix a column $\mathcal{C}$. For a suitable $\bar{x} \in \mathbb{R}^{n-1}$, the cubes $Q_{\nu}$ in $\mathcal{C}$ have centers $\left(\bar{x}, t_{1}\right), \ldots\left(\bar{x}, t_{N}\right)$, where $t_{1}, \ldots t_{N}$ form an arithmetic progression with the step $c \delta$. For each $i(1 \leq i \leq N)$, we have $\left(\bar{x}, t_{i}\right) \in Q_{\nu} \subset Q^{0} \subset B(0,1 / 2)$.

Therefore, for $\tau \in[-\delta, \delta]$ and $i=1, \ldots, N$, we have

$$
t_{i}+\tau \in I,
$$

where $I$ is the interval $\{t \in \mathbb{R}:(\bar{x}, t) \in B(0,1)\}$.

Let $Q_{\nu}$ be one of the cubes in $\mathcal{C}$, with center $\left(\bar{x}, t_{i}\right)$. By definition, $Q_{\nu}$ carries the label $\left(i_{0}, \beta\right)$ (with $i_{0}=n$ ) if and only if $\partial^{\beta} F\left(\bar{x}, t_{i}+\tau\right)=0$ for some $\tau \in[-\delta, \delta]$. In view of (4.18), it follows that the number of $Q_{\nu} \in \mathcal{C}$ that carry the label $\left(i_{0}, \beta\right)$ is equal to the number of $t_{i}(i=1, \ldots, N)$ that lie within the distance $\delta$ from a zero of the function $g(t)=\partial^{\beta} F(\bar{x}, t)$, defined for $t \in I$. Hence, to prove Lemma 6 , it is enough to show:

There are at most $C$ distinct $i(i=1, \ldots N)$, such that $t_{i}$ lies within the distance $\delta$ from a zero of $g(t)(t \in I)$. 
Moreover, since $t_{1}, \ldots, t_{N}$ form an arithmetic progression with the step $c \delta$, assertion (4.19) will follow, if we can prove that

$$
\text { the function } g \text { has at most } C \text { distinct zeroes in } I \text {. }
$$

Thus, Lemma 6 is reduced to the task of proving (4.20).

For $t \in I$, we have $(\bar{x}, t) \in B(0,1)$ by definition of $I$, and therefore

$$
\left(\frac{d}{d t}\right)^{m-1-|\beta|} g(t)=\left(\frac{\partial}{\partial x_{n}}\right)^{m-1-|\beta|} \partial^{\beta} F(\bar{x}, t) \neq 0,
$$

thanks to the hypothesis (1) of Theorem 2. That is,

$$
\left(\frac{d}{d t}\right)^{m-1-|\beta|} g(t) \text { vanishes nowhere on } I .
$$

A standard argument, repeatedly applying Rolle's theorem from elementary calculus, shows that any function satisfying (4.21) can have at most $m-1-|\beta|$ distinct zeroes in $I$. Hence, (4.20) holds, completing the proof of Lemma 6.

\section{Conclusion}

We retain the notation and the setting of Section 4 .

Let $c_{1}$ be a small enough controlled constant, and suppose we are given $x \in V(F, \delta)$ with $0<\delta<c_{1}$. By definition of $V(F, \delta)$, we have $x \in B\left(0, c_{1}\right)$, and $\left|x-x^{0}\right|<\delta$ for some $x^{0} \in V(F)$. In particular, $x^{0} \in B\left(0, c_{1}+\delta\right) \subset$ $B\left(0,2 c_{1}\right) \subset Q^{0}$, so $x^{0} \in Q_{\nu}$ for some $\nu$. Thus, $Q_{\nu}$ contains a point of $V(F)$, and $\left|x-x_{\nu}\right| \leq\left|x-x^{0}\right|+\left|x^{0}-x_{\nu}\right|<\delta+$ diameter $Q_{\nu}=(1+\bar{c}) \delta$, i.e., $x \in B\left(x_{\nu},(1+\bar{c}) \delta\right)$. We have therefore proven the following:

For $0<\delta<c_{1}$, the set $V(F, \delta)$ is contained in the union of the balls $B\left(x_{\nu},(1+\bar{c}) \delta\right)$ over all $\nu$ such that $Q_{\nu}$ contains a point of $V(F)$.

From Section 4 (conclusion (4.17) and lemma 6), we see that there are at most $C \delta^{-(n-1)}$ distinct $\nu$ such that $Q_{\nu}$ contains a point of $V(F)$. Since each $B\left(x_{\nu},(1+\bar{c}) \delta\right)$ has volume $C \delta^{n}$, it follows from (5.1) that

$$
\operatorname{Vol}\{V(F, \delta)\} \leq C_{2} \delta \quad \text { for } 0<\delta<c_{1},
$$

where $C_{2}$ is a controlled constant. Estimate (5.2) is precisely the conclusion of Theorem 2. We recall from Section 2 that Theorem 1 follows from Theorem 2. Hence, the proofs of Theorems 1 and 2 are complete. 


\section{References}

[1] Bálint, P. Chernov, N., SzÁsz, D. And Tóth, I. P.: Geometry of multi-dimensional dispersing billiards. In Geometric methods in dynamics (I), 189-150. Astérisque 286 (2003).

Recibido: 7 de junio de 2005

Pavel Batchourine

Department of Mathematics

Princeton University

Princeton, NJ, 08544, USA

pbatchou@math.princeton.edu

Charles Fefferman

Department of Mathematics

Princeton University

Princeton, NJ, 08544, USA

cf@math.princeton. edu

The work of the second author was partially supported by NSF grants DMS-0245242 and DMS-0070692. 